\title{
MAXIMIZING THE EXPECTED DURATION OF OWNING A RELATIVELY BEST OBJECT IN A POISSON PROCESS WITH RANKABLE OBSERVATIONS
}

\author{
AIKO KURUSHIMA, ${ }^{*}$ Tokyo University of Science \\ KATSUNORI ANO, ${ }^{* *}$ Institute of Applied Mathematics
}

\begin{abstract}
Suppose that an unknown number of objects arrive sequentially according to a Poisson process with random intensity $\lambda$ on some fixed time interval $[0, T]$. We assume a gamma prior density $G_{\lambda}(r, 1 / a)$ for $\lambda$. Furthermore, we suppose that all arriving objects can be ranked uniquely among all preceding arrivals. Exactly one object can be selected. Our aim is to find a stopping time (selection time) which maximizes the time during which the selected object will stay relatively best. Our main result is the following. It is optimal to select the $i$ th object that is relatively best and arrives at some time $s_{i}^{(r)}$ onwards. The value of $s_{i}^{(r)}$ can be obtained for each $r$ and $i$ as the unique root of a deterministic equation.

Keywords: Optimal stopping problem; secretary problem; Poisson arrival; duration problem
\end{abstract}

2000 Mathematics Subject Classification: Primary 60G40

Secondary 62L15

\section{Introduction}

Our problem is as follows. We must select one object from an unknown number of rankable objects by some fixed finite time $T$. The objects are observed sequentially and appear according to a Poisson process with unknown intensity $\lambda$. Let $X_{i}$ be the relative rank of the $i$ th object, and set $X_{i}=1,2, \ldots$ when the $i$ th object is the relatively best, second best, third best, etc., respectively. Each ordering of the objects is supposed to be equally likely. Then the distribution of $X_{i}$ is $\mathrm{P}\left(X_{i}=j\right)=1 / i$ for $i=1,2, \ldots$ and $j=1,2, \ldots, i$. We assume that the intensity $\lambda$ of the Poisson arrival process $\{N(t)\}_{t \geq 0}$ has a gamma prior density $G_{\lambda}(r, 1 / a)$, where $r \in \mathbb{N}$ and $a>0$. We want to find a stopping time that maximizes the time during which the selected object will stay relatively best.

Our secretary problem is a combination of the duration problem with the Poisson arrival setting. For further information about the secretary problem, see [6]. The duration of owning a relatively best object is studied by Ferguson et al. [8]. They found optimal stopping times for many interesting versions of the duration problem in the 'no-information' and 'full-information' cases, for the problems treated in [10] and [11] with a random number of objects, and for the Poisson arrival models studied in [2] and [5]. Here we say no information if we can observe only the relative rank of the objects and if each ordering of the objects occurs equally likely. We

Received 23 May 2007; revision received 23 March 2009.

* Postal address: Department of Industrial Management and Engineering, Faculty of Engineering, Tokyo University of Science, 1-14-6 Kudan-kita, Chiyoda-ku, Tokyo, 102-0073, Japan. Email address: kul@ ms.kagu.tus.ac.jp

** Postal address: Department of Applied Probability, Asakusabashi, Taito-ku, Tokyo, Japan.

Email address: kano@iapm.jp 
speak of full information if we can observe the value of each object, assumed to be independent and identically distributed from a known distribution. The optimal stopping time for Ferguson et al.'s problem with exponential prior intensity $G_{\lambda}(1,1 / a), a>0$, is given by

$$
\tau_{1}^{*}=\min \left\{t \in\left[s^{(1)}, T\right]: X_{N(t)}=1\right\},
$$

where $s^{(1)}=\left[(T+a) / \mathrm{e}^{2}-a\right]^{+}$and $[x]^{+}=\max \{x, 0\}$. It is then optimal to select the relatively best object which appears from time $s^{(1)}$ onwards. The authors have also shown that, for $a$ close to 0 (large arrival rate), the optimal expected duration time is close to $2 \mathrm{e}^{-2} T$.

Cowan and Zabczyk [5] assumed that the intensity of the process was known. The aim was to maximize the probability of selecting the best object overall. Bruss [2] succeeded in extending the problem to unknown intensities having exponential prior density $G_{\lambda}(1,1 / a), a>0$, and in finding the optimal stopping time. It is interesting to see that it has the same form as the optimal stopping time of Ferguson et al.'s duration problem (1) with the different value of $s^{(1)}=[(T+a) / \mathrm{e}-a]^{+}$. Ano [1] extended this problem to the problem of the unknown intensity having a gamma prior intensity $G_{\lambda}(r, 1 / a), r \in \mathbb{N}$, and found the optimal stopping time for $r=2$. Kurushima and Ano [9] found the optimal stopping time for the cases in which $r=3,4, \ldots$. Furthermore, the game version of Bruss' problem has been studied in [13].

As we have seen above, our problem can be considered as an extension of both [2] and [8]. The main result of this paper is the proof that the optimal stopping time $\tau_{r}^{*}$ is a threshold stopping time:

$$
\tau_{r}^{*}=\min \left\{t \in\left[s_{i}^{(r)}, T\right]: X_{i}=1 \text { for each } i=1,2,3, \ldots\right\},
$$

where $s_{i}^{(r)}$ is the unique root of a deterministic equation for each $r$ and $i$. It is optimal to select the $i$ th object to arrive provided it is relatively best and appears from time $s_{i}^{(r)}$ onwards. For each $r$, the threshold sequence $\left\{s_{i}^{(r)}\right\}_{i \geq 1}$ is nonincreasing. Furthermore, we see that the asymptotic value $s_{i}^{(r)}$ converges to $\left[(T+a) / \mathrm{e}^{2}-a\right]^{+}$as $i \rightarrow \infty$, which means that $N(T) \rightarrow \infty$ almost surely. Roughly speaking, when a large number of objects arrive in $[0, T]$, it is almost optimal to select the first relatively best object arriving after $\left[(T+a) / \mathrm{e}^{2}-a\right]^{+}$.

The paper is organized as follows. In Section 2 we formulate the reward process. Our formulation is similar to that of [1]. We study the cases in which $r=1,2$, and 3. In Section 3 we prove the main result.

\section{Formulation}

\subsection{Stopping reward}

Let $S_{1}, S_{2}, \ldots$ denote the arrival times of the Poisson process $\{N(t)\}_{t \geq 0}$. We assume that it has an unknown intensity $\lambda$ with gamma prior density

$$
g(\lambda)=\frac{a^{r} \mathrm{e}^{-a \lambda} \lambda^{r-1}}{\Gamma(r)} \mathbf{1}(\lambda \geq 0),
$$

where $r \in \mathbb{N}$ and $a>0$. By Bayes' theorem, the posterior density of $\lambda$ given $S_{1}=s_{1}, \ldots, S_{i}=$ $s$ can be computed and also turns out to be gamma, $G_{\lambda}(r+1,1 /(a+s))$. Bruss [2] showed that after observing $S_{1}=s_{1}, \ldots, S_{i}=s, 0<s<T$, the posterior distribution of $N(T)$ depends only on the values of $i$ and $S_{i}$, and is a Pascal distribution with parameters $(r+1,(s+a) /(T+a))$. Indeed, it was later shown that this is a special case of a Pascal process (see [3]).

Let $(i, s)$ denote the state of the process when the $i$ th object arrives at time $s$ and is relatively best. Note that we can confine our interest to such states of the decision process. Recall that 
$X_{i}$ is the relative rank of the $i$ th object. Let $p_{(i, s)}^{(k, u)}$ denote the transition probability from state $(i, s)$ to $(i+k, s+u)$, which, as Ano [1] (or Kurushima and Ano [9]) showed, is given by

$$
\begin{aligned}
p_{(i, s)}^{(k, u)}= & \int_{0}^{\infty} \mathrm{P}\left(S_{i+k}=s+u \mid S_{i}=s, \lambda\right) \mathrm{P}\left(X_{i+k}=1 \mid X_{i}=1, S_{i}=s, S_{i+k}=s+u, \lambda\right) \\
& \quad \times g\left(\lambda \mid S_{i}=s\right) \mathrm{d} \lambda \\
= & \frac{\Gamma(i+k+r)}{\Gamma(k) \Gamma(i+r)(i+k)(i+k-1)} \frac{i}{(i+k-1)(i+k)} \frac{s+a}{(s+a+u)^{2}} \\
& \times\left(\frac{s+a}{s+a+u}\right)^{i+r-1}\left(\frac{u}{s+a+u}\right)^{k-1} .
\end{aligned}
$$

The stopping reward is the expected duration time possessing the relatively best object when we stop at state $(i, s)$. Denote the expected duration time when we stop at state $(i, s)$ by $y_{i}^{(r)}(s)$. Using Bruss' result and (2), we have

$$
\begin{aligned}
y_{i}^{(r)}(s)= & \mathrm{E}(u \mid(i, s))+(T-s) \mathrm{P}(\text { no relatively best appears in }(s, T] \mid(i, s)) \\
= & \int_{0}^{\infty} u \sum_{k \geq 1} p_{(i, s)}^{(k, u)} \mathrm{d} u+(T-s) \sum_{n \geq i}\left(\frac{i}{n}\right) \mathrm{P}\left(N(T)=n \mid S_{i}=s\right) \\
= & \int_{0}^{T-s} \sum_{k \geq 1} \frac{(i+r+k-1) !}{(k-1) !(i+r-1) !} \frac{i}{(i+k)(i+k-1)} x^{i+r}(1-x)^{k} \mathrm{~d} u \\
& +(T-s) \sum_{n \leq i}\left(\frac{i}{n}\right) \frac{(n+r-1) !}{(r+i-1) !(n-i-1) !} \theta^{r+i}(1-\theta)^{n-i},
\end{aligned}
$$

where $x=(s+a) /(s+a+u)$ and $\theta=(s+a) /(T+a)$.

Using (2) and the identities

$$
\begin{array}{cc}
\frac{(n+r-1) !}{n}=(r-1) ! \sum_{i=0}^{r-1} \frac{(n+i-1) !}{i !}, & r=1,2, \ldots, \\
1=\sum_{k \geq 1} \frac{(n+k-1) !}{(k-1) ! n !} x^{n+1}(1-x)^{k-1}, & 0 \leq x \leq 1,
\end{array}
$$

we can calculate the expected duration time $y_{i}^{(r)}(s)$ as follows:

$$
\begin{aligned}
y_{i}^{(r)}(s)= & \left(\begin{array}{c}
i+r-1 \\
r-1
\end{array}\right)^{-1} \int_{0}^{T-s} \sum_{k \geq 1} \sum_{j=0}^{r-1} \frac{(i+k+j-1) ! x^{i+r}(1-x)^{k}}{(i-1) !(k-1) ! j !(i+k-1)} \mathrm{d} u \\
& +\frac{i(r-1) !(T-s)}{(i+r-1) !} \sum_{j=0}^{r-1} \sum_{k \geq 1} \frac{(n+j-1) !}{(n-i) !} \theta^{r+i}(1-\theta)^{n-i} \\
= & \left(\begin{array}{c}
i+r-1 \\
r-1
\end{array}\right)^{-1} \int_{0}^{T-s} \sum_{j=0}^{r-1} \sum_{l=0}^{j}\left(\begin{array}{c}
i+l-1 \\
l
\end{array}\right) \sum_{k \geq 1}\left(\begin{array}{c}
i+k+l-2 \\
k-1
\end{array}\right) x^{r+i}(1-x)^{n-i} \mathrm{~d} u \\
& +(T-s)\left(\begin{array}{c}
i+r-1 \\
r-1
\end{array}\right)^{-1} \sum_{j=0}^{r-1}\left(\begin{array}{c}
i+j-1 \\
j
\end{array}\right) \theta^{r-j} .
\end{aligned}
$$


A straightforward calculation using identity (4) and setting $x=(s+a) /(s+a+u)$ shows that the first terms on the right-hand side of (5) become

$$
\begin{aligned}
&(s+a)\left(\begin{array}{c}
i+r-1 \\
r-1
\end{array}\right)^{-1} \int_{(s+a) /(T+a)}^{1} \sum_{j=0}^{r-1} \sum_{l=0}^{j}\left(\begin{array}{c}
i+l-1 \\
l
\end{array}\right) x^{r-l}(1-x) \mathrm{d} x \\
&=(s+a)\left(\begin{array}{c}
i+r-1 \\
r-1
\end{array}\right) \int_{\theta}^{-1}\left\{\left(\begin{array}{c}
i+r-2 \\
r-1
\end{array}\right) x^{-1}+\sum_{l=0}^{r-1}\left(\begin{array}{c}
i+l-1 \\
l
\end{array}\right) x^{r-l-2}\right\} \mathrm{d} x \\
&+(s+a)\left(\begin{array}{c}
i+r-1 \\
r-1
\end{array}\right)^{-1} \int_{\theta}^{1}\left\{\sum_{j=0}^{r-2} \sum_{l=0}^{j}\left(\begin{array}{c}
i+l-1 \\
l
\end{array}\right) x^{r-l-2}\right. \\
&=(s+a)\left(\begin{array}{c}
i+r-1 \\
r-1
\end{array}\right)^{-1}\left\{\left(\begin{array}{c}
i+r-2 \\
r-1
\end{array}\right)(-\ln \theta)+\sum_{l=0}^{r-1} \sum_{j}^{j}\left(\begin{array}{c}
i+l-1 \\
l
\end{array}\right) x^{r-l-1}\right\} \mathrm{d} x \\
&+(s+a)\left(\begin{array}{c}
i+r-1 \\
r-1
\end{array}\right)^{-1}\left\{\sum_{l=0}^{r-2}\left(\begin{array}{c}
i+l-1 \\
l
\end{array}\right)\left(1-\theta^{r-l-1}\right)\right. \\
&\left.+\sum_{l=0}^{r-1}\left(\begin{array}{c}
i+l-1 \\
l
\end{array}\right)\left(1-\theta^{r-l}\right)\right\}
\end{aligned}
$$

Substituting (6) into (5), completes the proof of the following lemma.

Lemma 1. Let $y_{i}^{(r)}(s)$ be the stopping reward in state $(i, s)$, that is, the expected duration time when we stop at state $(i, s)$. Then,

$$
y_{i}^{(r)}(s)=(s+a)\left(\begin{array}{c}
i+r-1 \\
r-1
\end{array}\right)^{-1}\left\{\left(\begin{array}{c}
i+r-2 \\
r-1
\end{array}\right)(-\ln \theta)+\sum_{j=0}^{r-2}\left(\begin{array}{c}
i+j-1 \\
j
\end{array}\right) \frac{1-\theta^{r-j-1}}{r-j-1}\right\},
$$

where $\theta=(s+a) /(T+a)$.

\subsection{Monotone property of the stopping problem}

First we shortly summarize the monotone stopping problem based on Chow et al. [4, pp. 5455]. Let $\left\{Y_{i}\right\}_{i \geq 1}$ be the stopping reward process. If the process is supermartingale then the stopping problem is said to be monotone. Let the one-stage look-ahead (OLA) stopping time be denoted by $\tau=\min \left\{i \geq 1: Y_{i} \geq \mathrm{E}\left(Y_{i+1} \mid \mathcal{F}_{i}\right)\right\}$. Let the OLA stopping region be denoted by $B=\left\{i: Y_{i} \geq \mathrm{E}\left(Y_{i+1} \mid \mathcal{F}_{i}\right)\right\}$. If $B$ is 'closed', that is, $\mathrm{P}(j \in B \mid i \in B)=1$ for $j=i+1, i+2, \ldots$, then the problem is monotone and $\tau$ is optimal. Throughout the paper, we use the term 'closed' in this sense. This monotone property also holds for the reward process related to a stationary Markov chain (see Chapter 5 of [4], [7], or Chapter 6 of [12]).

We shall obtain the OLA stopping region for our problem and prove that it is closed. For each $r=1,2, \ldots$, let

$$
H_{i}^{(r)}(s)=\left(\begin{array}{c}
i+r-1 \\
r-1
\end{array}\right)(s+a)^{-1}\left\{y_{i}^{(r)}(s)-\int_{0}^{\infty} \sum_{k \geq 1} p_{(i, s)}^{(k, u)} y_{i+k}^{(r)}(s+u) \mathrm{d} u\right\} .
$$


Then we have the OLA stopping time $\tau_{r}$ and the OLA stopping region $B_{r}$,

$$
\tau_{r}=\min \left\{i \geq 1: H_{i}^{(r)}(s) \geq 0\right\} \quad \text { and } \quad B_{r}=\left\{(i, s): H_{i}^{(r)}(s) \geq 0\right\} .
$$

Now we calculate $H_{i}^{(r)}(s)$. From Lemma 1 we have

$$
\begin{aligned}
\int_{0}^{\infty} \sum_{k \geq 1} p_{(i, s)}^{(k, u)} y_{i+k}^{(r)}(s+u) \mathrm{d} u \\
=\int_{0}^{\infty} \sum_{k \geq 1} \frac{(i+r+k-2) !}{(k-1) !(i+r-1) !} \frac{i x^{i+r}(1-x)^{k-1}(-\ln \tilde{\theta})}{i+k-1} \mathrm{~d} u \\
\quad+\int_{0}^{\infty} \sum_{k \geq 1} \frac{i(r-1) ! x^{i+r}(1-x)^{k-1}}{(k-1) !(i+r-1) !} \sum_{j=0}^{r-2} \frac{(i+k+j-1) !\left(1-\tilde{\theta}^{r-j-1}\right)}{j !(i+k-1)(r-j-1)} \mathrm{d} u,
\end{aligned}
$$

where $\tilde{\theta}=(s+a+u) /(T+a)$. Using identities (3) and (4) again and calculating in a similar way to $y_{i}^{(r)}(s)$, we have

$$
\begin{aligned}
\int_{0}^{\infty} \sum_{k \geq 1} p_{(i, s)}^{(k, u)} y_{i+k}^{(r)}(s+u) \mathrm{d} u \\
=-\left(\begin{array}{c}
i+r-2 \\
r-1
\end{array}\right) \frac{1}{2}(\ln \theta)^{2}-\sum_{j=0}^{r-2}\left(\begin{array}{c}
i+j-1 \\
j
\end{array}\right) \frac{1-\theta^{r-j-1}}{r-j-1} \ln \theta \\
\quad+\sum_{j=1}^{r-2} \sum_{l=0}^{j-1}\left(\begin{array}{c}
i+l-1 \\
l
\end{array}\right) \frac{(j-l)+(r-j-1) \theta^{r-l-1}-(r-l-1) \theta^{r-j-1}}{(j-l)(r-j-1)(r-l-1)}
\end{aligned}
$$

Substituting the above equation into (7), we obtain

$$
\begin{aligned}
H_{i}^{(r)}(s) \equiv & h_{i}^{(r)}(\theta) \\
= & -\left(\begin{array}{c}
i+r-2 \\
r-1
\end{array}\right) \ln \theta\left(1+\frac{1}{2} \ln \theta\right)+\sum_{j-0}^{r-2}\left(\begin{array}{c}
i+j-1 \\
j
\end{array}\right) \frac{1-\theta^{r-j-1}}{r-j-1}(1+\ln \theta) \\
& -\sum_{j=1}^{r-2} \sum_{l=0}^{j-1}\left(\begin{array}{c}
i+l-1 \\
l
\end{array}\right) \frac{(j-l)+(r-j-1) \theta^{r-l-1}-(r-l-1) \theta^{r-j-1}}{(j-l)(r-j-1)(r-l-1)},
\end{aligned}
$$

where $\theta=(s+a) /(T+a) \in[0,1]$. Thus, for each $r, \tau_{r}$ and $B_{r}$ can be rewritten as

$$
\tau_{r}=\min \left\{i \geq 1: h_{i}^{(r)}(\theta) \geq 0\right\} \quad \text { and } \quad B_{r}=\left\{(i, \theta): h_{i}^{(r)}(\theta) \geq 0\right\} .
$$

\subsection{The cases in which $r=1,2$, and 3}

From (8), the functions $h_{i}^{(r)}$ for $r=1,2$, and 3 are respectively given by

$$
\begin{aligned}
& h_{i}^{(1)}(\theta)=-\ln \theta\left(1+\frac{\ln \theta}{2}\right), \\
& h_{i}^{(2)}(\theta)=-i \ln \theta\left(1+\frac{\ln \theta}{2}\right)+(1-\theta)(1+\ln \theta), \\
& h_{i}^{(3)}(\theta)=-\frac{i(i+1)}{2} \ln \theta\left(1+\frac{\ln \theta}{2}\right)+\frac{1-\theta^{2}}{2}(1+\ln \theta)+i(1-\theta)(1+\ln \theta)-\frac{(1-\theta)^{2}}{2} .
\end{aligned}
$$


Case 1: $r=1$. (See [8].) This is the same problem as that given in Section 4.1 of [8]. The function $h_{i}^{(1)}(\theta)$ does not depend on $i$ and so we may write it as $h^{(1)}(\theta)$. We can see that $h^{(1)}(\theta)$ is a unimodal function in $\theta \in(0,1]$ with $h^{(1)}(0+)=-\infty$ and $h^{(1)}(1)=0$, since $h^{(1)^{\prime}}(\theta)$ is increasing in $\theta \in\left(0, \mathrm{e}^{-1}\right], h^{(1)^{\prime}}(\theta)$ is decreasing in $\theta \in\left[\mathrm{e}^{-1}, 1\right]$, and $h^{(1)^{\prime \prime}}(\theta) \leq 0$ for $\theta \in(0,1]$. Therefore, $B_{1}$ can be written as

$$
B_{1}=\left\{\theta: h^{(1)}(\theta) \geq 0\right\}=\left\{\theta: \theta \geq \mathrm{e}^{-2}\right\}=\left\{s: s \geq\left[\frac{T+a}{\mathrm{e}^{2}}-a\right]^{+}\right\} .
$$

It is closed because once $h^{(1)}(\theta)$ becomes nonnegative then it stays nonnegative in $\left(\mathrm{e}^{-2}, 1\right]$. Thus, the optimal stopping time is given by (1).

Case 2: $r=2$. We first study the function $h_{i}^{(2)}(\theta)$ for each $i=1,2, \ldots$

Lemma 2. For $r=2$, the following statements hold.

(i) There exists a unique root $\theta_{i}^{(2)} \in\left(\mathrm{e}^{-2}, \mathrm{e}^{-1}\right)$ of the equation $h_{i}^{(2)}(\theta)=0$ for each $i=$ $1,2, \ldots$. Also, $h_{i}^{(2)}(\theta) \geq 0$ implies that $h_{i}^{(2)}(\theta+\eta) \geq 0$ for $0 \leq \eta \leq 1-\theta$.

(ii) For each $i=1,2, \ldots, h_{i}^{(2)}(\theta) \geq 0$ implies that $h_{i+1}^{(2)}(\theta) \geq 0$.

Proof. (i) For $\theta \in(0,1]$, we have $h_{i}^{(2) "}(\theta) \leq 0$, implying that $h_{i}^{(2)}(\theta)$ is a unimodal function with $h_{i}^{(2)}(0+)=\infty$ and $h_{i}^{(2)}(1)=0$. We also have $h_{i}^{(2)}\left(\mathrm{e}^{-2}\right)<0$ and $h_{i}^{(2)}\left(\mathrm{e}^{-1}\right)>0$. Therefore, (i) holds.

(ii) From (i), $h_{i}^{(2)}(\theta)$ changes sign once from negative to nonnegative in $\left[\mathrm{e}^{-2}, 1\right]$. On the other hand, for $\theta \in\left[\mathrm{e}^{-2}, 1\right]$,

$$
h_{i+1}^{(2)}(\theta)-h_{i}^{(2)}(\theta)=-\ln \theta\left(1+\frac{\ln \theta}{2}\right) \geq 0 .
$$

Thus, if $h_{i}^{(2)}(\theta) \geq 0$ then $h_{i+1}^{(2)}(\theta) \geq h_{i}^{(2)}(\theta) \geq 0$.

Theorem 1. If $r=2$, the optimal stopping time $\tau_{2}^{*}$ is given by $\tau_{2}^{*}=\min \left\{t \in\left[s_{i}^{(2)}, T\right]: X_{i}=1\right.$, $i=1,2, \ldots\}$, where $s_{i}^{(2)} \in(0, T]$ is the unique root of the equation $H_{i}^{(2)}(s)=0$ for each $i=1,2, \ldots$.

Proof. Lemma 2 implies the following.

(i) There exists a unique root $s_{i}^{(2)} \in(0, T]$ of the equation $H_{i}^{(2)}(\theta)=0$, and $H_{i}^{(2)}(s) \geq 0$ implies that $H_{i}^{(2)}(s+u) \geq 0$ for $0 \leq u \leq 1-s$.

(ii) For each $i=1,2, \ldots, H_{i}^{(2)}(s) \geq 0$ implies that $H_{i+1}^{(2)}(s) \geq 0$, or, equivalently, $H_{i}^{(2)}(s) \geq$ 0 implies that $H_{i+k}^{(2)}(s) \geq 0$ for $\bar{k}=1,2, \ldots$.

Therefore, $B_{2}$ is given by $B_{2}=\left\{(i, \theta): h_{i}^{(2)}(\theta) \geq 0\right\}=\left\{(i, s): H_{i}^{(2)}(s) \geq 0\right\}$ and it is closed. So the first hitting time $\tau_{2}^{*}$ for $B_{2}$ is the optimal stopping time given by $\tau_{2}^{*}=\min \{s \geq$ $\left.s_{i}^{(2)}:(i, s) \in B_{2}\right\}=\min \left\{t \in\left[s_{i}^{(2)}, T\right]: X_{i}=1, i=1,2, \ldots\right\}$.

Case 3: $r=3$.

Lemma 3. For $r=3$, the following statements hold.

(i) There exists a unique root $\theta_{i}^{(3)} \in\left(\mathrm{e}^{-2}, \mathrm{e}^{-1}\right)$ of the equation $h_{i}^{(3)}(\theta)=0$. Moreover, $h_{i}^{(3)}(\theta) \geq 0$ implies that $h_{i}^{(3)}(\theta+\eta) \geq 0$ for $0 \leq \eta \leq 1-\theta$.

(ii) For each $i=1,2, \ldots, h_{i}^{(3)}(\theta) \geq 0$ implies that $h_{i+1}^{(3)}(\theta) \geq 0$. 
Proof. (i) We have, for $\theta \in\left(0, \mathrm{e}^{-1}\right]$,

$$
\theta h_{i}^{(3)^{\prime}}(\theta)=-\left(\frac{i(i+1)}{2}+i \theta+\theta^{2}\right)(1+\ln \theta)+\frac{\left(1-\theta^{2}\right)}{2}+(i+\theta)(1-\theta) \geq 0,
$$

so that $h_{i}^{(3)}(\theta)$ is nondecreasing in $\theta \in\left(0, \mathrm{e}^{-1}\right]$. For $\theta \in\left[\mathrm{e}^{-1}, 1\right]$, we have

$$
\theta^{2} h_{i}^{(3)^{\prime \prime}}(\theta)=-\left(\theta^{2}-\frac{i(i+1)}{2}\right) \ln \theta-\frac{5}{2} \theta^{2}-i(1+\theta)-\frac{3}{2}<0,
$$

so that $h_{i}^{(3)}(\theta)$ is concave in $\theta \in\left(\mathrm{e}^{-1}, 1\right]$. Since $h_{i}^{(3)}(0+)=-\infty, h_{i}^{(3)}(1)=0, h_{i}^{(3)}\left(\mathrm{e}^{-2}\right)<0$, and $h_{i}^{(3)}\left(\mathrm{e}^{-1}\right)>0$, (i) holds.

(ii) By virtue of (i), if $h_{i+1}^{(3)}\left(\theta_{i}^{(3)}\right)>0$ then (ii) holds. There exists a unique $\operatorname{root} \theta_{i}^{(3)}$ in $\left(\mathrm{e}^{-2}, \mathrm{e}^{-1}\right)$, and so $1+\ln \theta_{i}^{(3)}<0$. From $h_{i}^{(3)}\left(\theta_{i}^{(3)}\right)=0$, the difference $h_{i+1}^{(3)}\left(\theta_{i}^{(3)}\right)-h_{i}^{(3)}\left(\theta_{i}^{(3)}\right)$ is equal to $h_{i+1}^{(3)}\left(\theta_{i}^{(3)}\right)$, which is given by

$$
h_{i+1}^{(3)}\left(\theta_{i}^{(3)}\right)=-(i+1) \ln \theta_{i}^{(3)}\left(1+\frac{\ln \theta_{i}^{(3)}}{2}\right)+\left(1-\theta_{i}^{(3)}\right)\left(1+\ln \theta_{i}^{(3)}\right) .
$$

On the other hand, $h_{i}^{(3)}\left(\theta_{i}^{(3)}\right)=0$ is equivalent to

$$
\begin{aligned}
-(i+1) \ln \theta_{i}^{(3)}\left(1+\frac{\ln \theta_{i}^{(3)}}{2}\right)= & -\frac{1-\theta_{i}^{(3)^{2}}}{i}\left(1+\ln \theta_{i}^{(3)}\right)-2\left(1-\theta_{i}^{(3)}\right)\left(1+\ln \theta_{i}^{(3)}\right) \\
& +\frac{\left(1-\theta_{i}^{(3)}\right)^{2}}{i} .
\end{aligned}
$$

Substituting the above equation into $h_{i+1}^{(3)}\left(\theta_{i}^{(3)}\right)$, we have

$$
h_{i+1}^{(3)}\left(\theta_{i}^{(3)}\right)=-\frac{1-\theta_{i}^{(3)^{2}}}{i}\left(1+\ln \theta_{i}^{(3)}\right)-\left(1-\theta_{i}^{(3)}\right)\left(1+\ln \theta_{i}^{(3)}\right)+\frac{\left(1-\theta_{i}^{(3)}\right)^{2}}{i} \geq 0,
$$

where the last inequality follows from $1+\ln \theta_{i}^{(3)}<0$. This completes the proof.

Theorem 2. For $r=3$, the optimal stopping time $\tau_{3}^{*}$ is given by $\tau_{3}^{*}=\min \left\{t \in\left[s_{i}^{(3)}, T\right]: X_{i}=\right.$ $1, i=1,2, \ldots\}$, where $s_{i}^{(3)} \in(0, T]$ is the unique root of the equation $H_{i}^{(3)}(s)=0$.

Proof. The proof is similar to that of Theorem 2 and is therefore omitted.

\section{Main results}

First we study the function $h_{i}^{(r)}(\theta)$ for each $i=1,2, \ldots$.

Proposition 1. For each $r=1,2, \ldots$ and $i=1,2, \ldots$,

(i) $h_{i}^{(r)}(\theta)$ is nondecreasing in $\theta \in\left(0, \mathrm{e}^{-1}\right]$,

(ii) $h_{i}^{(r)}(0+)=-\infty, h_{i}^{(r)}(1)=0$, and $h_{i}^{(r)}\left(\mathrm{e}^{-1}\right)>0$,

(iii) there exists a $\hat{\theta}$ such that $h_{i}^{(r)}(\theta)$ is nondecreasing in $\theta \in\left[\mathrm{e}^{-1}, \hat{\theta}\right]$ and nonincreasing in $\theta \in[\hat{\theta}, 1]$. 
Proof. It is hard to show these statements directly. We look at the behavior of $\theta h_{i}^{(r)}(\theta)$ instead of $h_{i}^{(r)}(\theta)$.

(i) We have

$$
\begin{aligned}
\theta h_{i}^{(r)^{\prime}}(\theta)= & -\left(\begin{array}{c}
i+r-2 \\
r-1
\end{array}\right)(1+\ln \theta)-\sum_{j=0}^{r-2}\left(\begin{array}{c}
i+j-1 \\
j
\end{array}\right) \theta^{r-j-1}(1+\ln \theta) \\
& +\sum_{j=0}^{r-2}\left(\begin{array}{c}
i+j-1 \\
j
\end{array}\right) \frac{1-\theta^{r-j-1}}{r-j-1}-\sum_{j=1}^{r-2} \sum_{l=0}^{j-1}\left(\begin{array}{c}
i+l-1 \\
l
\end{array}\right) \frac{\theta^{r-l-1}-\theta^{r-j-1}}{j-l} .
\end{aligned}
$$

As is easily shown, $(1+\ln \theta) \leq 0$ for $\theta \in\left(0, \mathrm{e}^{-1}\right]$ and $\theta^{r-l-1} \leq \theta^{r-j-1}$ for $\theta \in[0,1]$, from which it follows that $h_{i}^{(r)^{\prime}} \geq 0$ for $\theta \in\left(0, \mathrm{e}^{-1}\right]$.

(ii) It is easy to show that the first two statements hold. From (9) we have

$$
h_{i}^{(r)}\left(\mathrm{e}^{-1}\right)=\frac{1}{2}\left(\begin{array}{c}
i+r-1 \\
r-2
\end{array}\right)-f_{1}^{(r)}\left(\mathrm{e}^{-1}\right),
$$

where

$$
f_{1}^{(r)}(\theta)=\sum_{j=1}^{r-2} \sum_{l=0}^{j-1}\left(\begin{array}{c}
i+l-1 \\
l
\end{array}\right) \frac{(j-l)+(r-j-1) \theta^{r-l-1}-(r-l-1) \theta^{r-j-1}}{(j-l)(r-j-1)(r-l-1)} .
$$

The function $f_{1}^{(r)}(\theta)$, is a decreasing, nonnegative function on $[0,1]$, since $f_{1}^{(r)}(0)>0$, $f_{1}^{(r)}(1)=0$, and $f_{1}^{(r)^{\prime}}(\theta)<0$. Therefore, if we could show that

$$
\frac{1}{2}\left(\begin{array}{c}
i+r-1 \\
r-2
\end{array}\right)-f_{1}^{(r)}(0)>0
$$

then $h_{i}^{(r)}\left(\mathrm{e}^{-1}\right)>0$. We shall show this now.

Using identity (4) repeatedly, we have

$$
\frac{1}{2}\left(\begin{array}{c}
i+r-1 \\
r-2
\end{array}\right)=\frac{1}{2} \sum_{j=0}^{r-2}\left(\begin{array}{c}
i+j \\
j
\end{array}\right)=\frac{1}{2} \sum_{j=0}^{r-2} \sum_{l=0}^{j}\left(\begin{array}{c}
i+l-1 \\
l
\end{array}\right) .
$$

Then, for each $r=3,4, \ldots$,

$$
\begin{aligned}
\frac{1}{2}\left(\begin{array}{c}
i+ \\
r-2
\end{array}\right)-f_{1}^{(r)}(0) \\
=\left(\begin{array}{c}
i-1 \\
0
\end{array}\right)\left[\frac{1}{2}-\frac{1}{(r-2)(r-1)}\right] \\
\quad+\left(\begin{array}{c}
i-1 \\
0
\end{array}\right)\left[\frac{1}{2}-\frac{1}{(r-3)(r-1)}\right]+\left(\begin{array}{c}
i-2 \\
1
\end{array}\right)\left[\frac{1}{2}-\frac{1}{(r-3)(r-2)}\right]+\cdots \\
\quad+\left(\begin{array}{c}
i-1 \\
0
\end{array}\right)\left[\frac{1}{2}-\frac{1}{1(r-1)}\right]+\cdots+\left(\begin{array}{c}
i+r-4 \\
r-3
\end{array}\right)\left[\frac{1}{2}-\frac{1}{2}\right] \\
\quad+\frac{1}{2}\left\{\left(\begin{array}{c}
i-1 \\
0
\end{array}\right)+\left(\begin{array}{c}
i-2 \\
1
\end{array}\right)+\cdots+\left(\begin{array}{c}
i+r-3 \\
r-2
\end{array}\right)\right\} \\
>
\end{aligned}
$$


The last inequality follows since the differences in all the square brackets are nonnegative for each $r=3,4, \ldots$. For $r=1,2$, it is easy to show that $h^{(1)}\left(\mathrm{e}^{-1}\right)>0$ and $h_{i}^{(2)}\left(\mathrm{e}^{-1}\right)>0$, and, thus, the proof of (ii) is complete.

(iii) We want to prove that the function $h_{i}^{(r)^{\prime}}(\theta)$ changes sign once from negative to nonnegative in $(0,1)$. To show this, let $\theta h_{i}^{(r)^{\prime}}(\theta) \stackrel{=}{=}-f_{2}^{(r)}(\theta)+f_{3}^{(r)}(\theta)$, where $f_{2}^{(r)}(\theta)$ and $f_{3}^{(r)}(\theta)$ are given by

$$
\begin{aligned}
f_{2}^{(r)}(\theta)= & \left(\begin{array}{c}
i+r-2 \\
r-1
\end{array}\right)(1+\ln \theta)-\sum_{j=1}^{r-2} \sum_{l=0}^{j-1}\left(\begin{array}{c}
i+l-1 \\
l
\end{array}\right) \frac{\theta^{r-j-1}}{j-l}, \\
f_{3}^{(r)}(\theta)= & -\sum_{j=0}^{r-2}\left(\begin{array}{c}
i+j-1 \\
j
\end{array}\right)\left\{\theta^{r-j-1}(1+\ln \theta)-\frac{1-\theta^{r-j-1}}{r-j-1}\right\} \\
& -\sum_{j=1}^{r-2} \sum_{l=0}^{j-1}\left(\begin{array}{c}
i+l-1 \\
l
\end{array}\right) \frac{\theta^{r-l-1}}{j-l} .
\end{aligned}
$$

If

(a) $f_{3}^{(r)}\left(\mathrm{e}^{-1}\right)>f_{2}^{(r)}\left(\mathrm{e}^{-1}\right)$,

(b) $f_{2}^{(r)}(1)>f_{3}^{(r)}(1)$,

(c) $f_{2}^{(r)}(\theta)$ is a concave function for $\theta \in\left[\mathrm{e}^{-1}, 1\right]$, and

(d) $f_{3}^{(r)}(\theta)$ is a decreasing and concave function for $\theta \in\left[\mathrm{e}^{-1}, 1\right]$

hold, then there exists a unique root $\hat{\theta}$ in $\left[\mathrm{e}^{-1}, 1\right]$ of the equation $f_{2}^{(r)}(\theta)=f_{3}^{(r)}(\theta)$, or, equivalently, $h_{i}^{(r)^{\prime}}(\theta)=0$. This shows that the function $h_{i}^{(r)^{\prime}}(\theta)$ changes sign once from negative to nonnegative in $(0,1)$. For an example of $r=3$, see Figure 1 . We now prove conditions (a)-(d). Since $\mathrm{e}^{-(r-l-1)}<\mathrm{e}^{-(r-j-1)}$, we have

$$
f_{3}^{(r)}\left(\mathrm{e}^{-1}\right)-f_{2}^{(r)}\left(\mathrm{e}^{-1}\right)=\sum_{j=0}^{r-2} \frac{1-\mathrm{e}^{-(r-j-1)}}{r-j-1}-\sum_{j=1}^{r-2} \sum_{l=0}^{j-1} \frac{\mathrm{e}^{-(r-l-1)}-\mathrm{e}^{-(r-j-1)}}{j-l}>0 .
$$

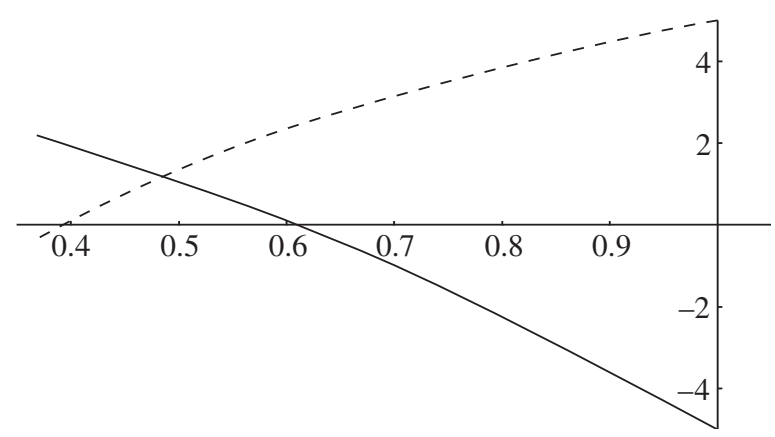

Figure 1: Graphs of $f_{2}^{(3)}(\theta)$ (dashed line) and $f_{3}^{(3)}(\theta)\left(\right.$ solid line) in $\left[\mathrm{e}^{-1}, 1\right]$. 


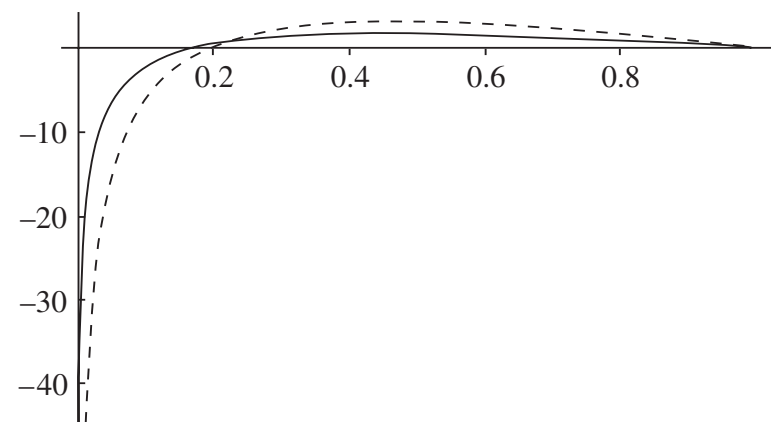

FIGURE 2: Graphs of $h_{2}^{(3)}(\theta)$ (dashed line) and $h_{3}^{(3)}(\theta)$ (solid line) in $(0,1]$.

Thus, (a) holds. Since

$$
-f_{2}^{(r)}(1)+f_{3}^{(r)}(1)=h_{i}^{(r)^{\prime}}(1)=-\left(\begin{array}{c}
i+r-2 \\
r-1
\end{array}\right)-\sum_{j=0}^{r-2}\left(\begin{array}{c}
i+j-1 \\
j
\end{array}\right)<0
$$

(b) holds. For $\theta \in\left[\mathrm{e}^{-1}, 1\right]$, a straightforward calculation yields $f_{2}^{(r)^{\prime \prime}}(\theta)<0, f_{3}^{(r)^{\prime}}(\theta)<0$, and $f_{3}^{(r)^{\prime \prime}}(\theta)<0$. Thus, (c) and (d) hold, and the proof is complete.

Now we have the following lemma.

Lemma 4. For each $r=1,2, \ldots$ and $i=1,2, \ldots$,

(i) there exists a unique root $\theta_{i}^{(r)}$ on $\left(0, \mathrm{e}^{-1}\right)$ of the equation $h_{i}^{(r)}(\theta)=0$,

(ii) $h_{i}^{(r)}(\theta) \geq 0$ implies that $h_{i}^{(r)}(\theta+\eta) \geq 0,0 \leq \eta \leq 1-\theta$,

(iii) $h_{i}^{(r)}(\theta) \geq 0$ implies that $h_{i+1}^{(r)}(\theta) \geq 0$.

Proof. Parts (i) and (ii) follow immediately from Proposition 1. Part (ii) states that once the function $h_{i}^{(r)}(\theta)$ becomes nonnegative, it remains nonnegative (for an example of $r=3$, see Figure 2). From (i) and (ii), for the proof of (iii), it suffices to show that $h_{i+1}^{(r)}\left(\theta_{i}^{(r)}\right)$ is nonnegative.

First, we have $h_{i}^{(r)}\left(\theta_{i}^{(r)}\right)=0$ implies that

$$
\begin{aligned}
& \left(\begin{array}{c}
i+r-2 \\
r-1
\end{array}\right) \ln \theta_{i}^{(r)}\left(1+\frac{\ln ^{2} \theta_{i}^{(r)}}{2}\right) \\
& \quad=\sum_{j=0}^{r-2}\left(\begin{array}{c}
i+j-1 \\
j
\end{array}\right) \frac{\left(1-\theta_{i}^{\left.(r)^{r-j-1}\right)\left(1+\ln \theta_{i}^{(r)}\right)}\right.}{r-j-1} \\
& \quad-\sum_{j=1}^{r-2} \sum_{l=0}^{j-1}\left(\begin{array}{c}
i+l-1 \\
l
\end{array}\right) \frac{f_{4}^{(r)}\left(\theta_{i}^{(r)}\right)}{(j-l)(r-j-1)(r-l-1)},
\end{aligned}
$$


where we set $f_{4}^{(r)}(\theta)=(j-l)+(r-j-1) \theta^{r-l-1}-(r-l-1) \theta^{r-j-1}$. Now note that

$$
\begin{aligned}
i\left(h_{i+1}^{(r)}(\theta)-h_{i}^{(r)}(\theta)\right)= & -(r-1)\left(\begin{array}{c}
i+r-2 \\
r-1
\end{array}\right) \ln \theta\left(1+\frac{\ln \theta}{2}\right) \\
& +\sum_{j=0}^{r-2} j\left(\begin{array}{c}
i+j-1 \\
j
\end{array}\right) \frac{1-\theta^{r-j-1}}{r-j-1}(1+\ln \theta) \\
& -\sum_{j=1}^{r-2} \sum_{l=0}^{j-1}\left(\begin{array}{c}
i+l-1 \\
l
\end{array}\right) \frac{l f_{4}^{(r)}(\theta)}{(j-l)(r-j-1)(r-l-1)} .
\end{aligned}
$$

Since $h_{i}^{(r)}\left(\theta_{i}^{(r)}\right)=0$, we have

$$
\begin{aligned}
i h_{i+1}^{(r)}\left(\theta_{i}^{(r)}\right)= & -(r-1)\left(\begin{array}{c}
i+r-2 \\
r-1
\end{array}\right) \ln \theta_{i}^{(r)}\left(1+\frac{\ln \theta_{i}^{(r)}}{2}\right) \\
& +\sum_{j=0}^{r-2} j\left(\begin{array}{c}
i+j-1 \\
j
\end{array}\right) \frac{1-\theta_{i}^{(r)}(-j-1}{r-j-1}\left(1+\ln \theta_{i}^{(r)}\right) \\
& -\sum_{j=1}^{r-2} \sum_{l=0}^{j-1}\left(\begin{array}{c}
i+l-1 \\
l
\end{array}\right) \frac{l f_{4}^{(r)}\left(\theta_{i}^{(r)}\right)}{(j-l)(r-j-1)(r-l-1)} .
\end{aligned}
$$

Substituting (10) into (11) we obtain

$$
\begin{aligned}
i h_{i+1}^{(r)}\left(\theta_{i}^{(r)}\right)= & -\sum_{j=0}^{r-2}\left(\begin{array}{c}
i+j-1 \\
j
\end{array}\right)\left(1-\theta_{i}^{(r)^{r-j-1}}\right)\left(1+\ln \theta_{i}^{(r)}\right) \\
& +\sum_{j=1}^{r-2} \sum_{l=0}^{j-1}\left(\begin{array}{c}
i+l-1 \\
l
\end{array}\right) \frac{(r-l-1) f_{4}^{(r)}\left(\theta_{i}^{(r)}\right)}{(j-l)(r-j-1)(r-l-1)} .
\end{aligned}
$$

From Proposition 1 we already know that $\theta_{i}^{(r)} \leq \mathrm{e}^{-1}$ and, hence, $1+\ln \theta_{i}^{(r)}<0$. Recall that $f_{4}^{(r)}(\theta) \geq 0$ for all $\theta \in[0,1]$. Then the right-hand side of (12) is positive, which shows that $h_{i+1}^{(r)}\left(\theta_{i}^{(r)}\right)>0$. This completes the proof.

We now state and prove the main result.

Theorem 3. For a Poisson process with gamma prior intensity $G_{\lambda}(r, 1 / a), a>0$ and $r=$ $1,2, \ldots$, the optimal stopping time $\tau_{r}^{*}$ maximizing the expected duration time owning a relatively best object is given by $\tau_{r}^{*}=\min \left\{t \in\left[s_{i}^{(r)}, T\right]: X_{i}=1, i=1,2, \ldots\right\}$, where $s_{i}^{(r)}$ is the unique root of the equation $H_{i}^{(r)}(s)=0$ in $[0, T]$ for each $r$ and $i$, and $H_{i}^{(r)}(s)$ is given by (8).

Proof. We have $B_{r}=\left\{(i, s): H_{i}^{(r)}(s) \geq 0\right\}=\left\{(i, \theta): h_{i}^{(r)}(\theta) \geq 0\right\}$. Lemma 4 directly shows that $B_{r}$ is closed and the problem becomes monotone. Therefore, $B_{r}$ is the optimal stopping region and the OLA stopping time $\tau_{r}^{*}$ is optimal.

Now we shall see the characteristic of the threshold values precisely.

Proposition 2. For each $r=1,2, \ldots$ and $i=1,2, \ldots$, there exists a unique root $\theta_{i}^{(r)} \in$ $\left(\mathrm{e}^{-2}, \mathrm{e}^{-1}\right)$ of the equation $h_{i}^{(r)}(\theta)=0$. 
Proof. We have

$$
\begin{aligned}
h_{i}^{(r)}\left(\mathrm{e}^{-2}\right)= & -\sum_{j=0}^{r-2}\left(\begin{array}{c}
i+j-1 \\
j
\end{array}\right) \frac{1-\mathrm{e}^{-2(r-j-1)}}{(r-j-1)} \\
& -\sum_{j=1}^{r-2} \sum_{l=0}^{j-1}\left(\begin{array}{c}
i+l-1 \\
l
\end{array}\right) \frac{f_{4}^{(r)}\left(\mathrm{e}^{-2}\right)}{(j-l)(r-j-1)(r-l-1)},
\end{aligned}
$$

where we set $f_{4}^{(r)}(\theta)=(j-l)+(r-j-1) \theta^{r-l-1}-(r-l-1) \theta^{r-j-1}$. Since $f_{4}^{(r)}(0)>0$, $f_{4}^{(r)}(1)=0$, and $f_{4}^{(r)^{\prime}}(\theta) \leq 0$, we have $f_{4}^{(r)}(\theta)>0$ for $\theta \in[0,1)$. Therefore, $f_{4}^{(r)}\left(\mathrm{e}^{-2}\right)>0$, which yields $h_{i}^{(r)}\left(\mathrm{e}^{-2}\right)<0$.

Theorem 4. The threshold sequence has the following properties.

(i) For each $r=1,2, \ldots$, the threshold sequence $\left\{s_{i}^{(r)}\right\}_{i \geq 1}$ is nonincreasing.

(ii) The limiting value of the threshold $s_{i}^{(r)}$ for all $r=1,2, \ldots$ is given by $\lim _{i \rightarrow \infty} s_{i}^{(r)}=$ $\left[(T+a) / \mathrm{e}^{2}-a\right]^{+}$.

Proof. (i) The proof follows immediately from Lemma 4.

(ii) Since $s_{i}^{(r)}=\theta_{i}^{(r)}(T+a)-a$, it suffices to prove that $\lim _{i \rightarrow \infty} \theta_{i}^{(r)}=\mathrm{e}^{-2}$. When $h_{i}^{(r)}(\theta)=0$, we have

$$
\begin{aligned}
-\ln \theta\left(1+\frac{\ln \theta}{2}\right)= & \left(\begin{array}{c}
i+r-2 \\
r-1
\end{array}\right) \sum_{j=0}^{-1}\left(\begin{array}{c}
i+j-1 \\
j
\end{array}\right) \frac{\left(1-\theta^{r-j-1}\right)(1+\ln \theta)}{r-j-1} \\
& +\left(\begin{array}{c}
i+r-2 \\
r-1
\end{array}\right) \sum_{j=1}^{-1} \sum_{l=0}^{r-2}\left(\begin{array}{c}
i+l-1 \\
l
\end{array}\right) \frac{f_{4}^{(r)}(\theta)}{(j-l)(r-j-1)(r-l-1)}
\end{aligned}
$$

It is easy to check that, as $i$ tends to $\infty$,

$$
\left(\begin{array}{c}
i+r-2 \\
r-1
\end{array}\right) \sum_{j=1}^{-1}\left(\begin{array}{c}
i+j-1 \\
j
\end{array}\right)=\frac{(r-1) !}{(i+r-2) \cdots i}+\cdots+\frac{r-1}{i+r-2} \rightarrow 0
$$

and

$$
\begin{aligned}
\left(\begin{array}{c}
i+r-2 \\
r-1
\end{array}\right) \sum_{j=1}^{-1} \sum_{l=0}^{r-2}\left(\begin{array}{c}
i+l-1 \\
l
\end{array}\right)= & \left\{\frac{(r-1) !}{(i+r-2) \cdots i}+\cdots+\frac{(r-1) !}{(i+r-2) \cdots i}\right\} \\
& +\cdots+\left\{\frac{(r-1) !}{(i+r-2) \cdots i}+\cdots+\frac{(r-1)(r-2)}{(i+1) !}\right\} \\
\rightarrow & 0 .
\end{aligned}
$$

Therefore, the right-hand side of (13) goes to 0 and $-\ln \theta(1+(\ln \theta / 2)) \rightarrow 0$ as $i \rightarrow \infty$. Hence, $\lim _{i \rightarrow \infty} \theta_{i}^{(r)}=\mathrm{e}^{-2}$, which completes the proof. 


\section{Acknowledgements}

We are grateful to the anonymous referee for his/her constructive comments and suggestions, which led to substantial improvements in the exposition and a reconsideration of the meaning of the threshold values, correcting our description of the optimal stopping time. The second author is grateful to Professor Thomas Ferguson for his suggestion of using identities (3) and (4) to obtain the stopping reward for the Poisson arrival type stopping problem with unknown intensity. Without it, we could not have calculated the stopping reward.

\section{References}

[1] Ano, K. (2000). A Poisson arrival selection problem for Gamma prior intensity with parameter $r=2$. In Proc. Internat. Conf. Appl. Stoch. System Modeling (Kyoto, 2000), pp. 10-17.

[2] Bruss, F. T. (1987). On an optimal selection problem by Cowan and Zabczyk. J. Appl. Prob. 24, $918-928$.

[3] Bruss, F. T. And Rogers, L. C. G. (1991). Pascal processes and their characterization. Stoch. Proc. Appl. 37, 331-338.

[4] Chow, Y. S., Robbins, H. And Siegmund, D. (1971). Great Expectations: The Theory of Optimal Stopping. Houghton Mifflin, Boston, MA.

[5] Cowan, R. And ZabCZYK, J. (1978). An optimal selection problem associated with the Poisson process. Theory Prob. Appl. 23, 606-614.

[6] Ferguson, T. S. (1989). Who solved the secretary problem? Statist. Sci. 4, 282-296.

[7] Ferguson, T. S. (1992). Optimal Stopping and Applications. Available at http://www.math.ucla.edu/ tom/ Stopping/Contents.html.

[8] Ferguson, T. S., Hardwick, J. P. and Tamaki, M. (1992). Maximizing the duration of owning a relatively best object. In Strategies for Sequential Search and Selection in Real Time (Amherst, MA, (1990); Contemp. Math. 125), American Mathematical Society, Providence, RI, pp. 37-57.

[9] Kurushima, A. And Ano, K. (2003). A Poisson arrival selection problem for gamma prior intensity with natural number parameter. Sci. Math. Japonica 57, 217-231.

[10] Porosinski, A. (1987). The full-information best choice problem with a random number of observations. Stoch. Process. Appl. 24, 293-307.

[11] Presman, È. L. And Sonin, I. M. (1972). The problem of best choice in the case of a random number of objects. Theory Prob. Appl. 17, 695-706.

[12] Ross, S. M. (1970). Applied Probability Models and Optimization Applications. Holden-Day, San Francisco, CA.

[13] Szajowski, K. (2007). A game version of the Cowan-Zabczyk-Bruss' problem. Statist. Prob. Lett. 77, 16831689. 